\title{
Diferença entre dois métodos de coleta utilizados na captura de crustáceos decápodes em um rio da Estação Ecológica Juréia-Itatins, São Paulo
}

\author{
Sérgio Schwarz da Rocha
}

Centro de Ciências Agrárias, Ambientais e Biológicas, Universidade Federal do Recôncavo da Bahia, 44380-000 Cruz das Almas, BA, Brasil. (ssrocha@ufrb.edu.br)

\begin{abstract}
Differences between two methods used for sampling decapod crustaceans in a stream at Juréia-Itatins Ecological Station, state of São Paulo, Brazil. Sampling collections were taken quarterly from Fall 2000 to Summer 2001 at JuréiaItatins Ecological Station using two different sampling methods (hand sieve and traps). Comparing both sampling methods one can verify differences in faunal composition, richness, degree of dominance, evenness and size and number of individuals. Moreover, the sex ratio of Macrobrachium acanthurus (Wiegmann, 1836) may differ according to the sampling method. Decapod crustaceans are known to inhabit a great variety of habitats and niches, therefore to obtain an effective qualitative and/or quantitative sampling, in which the majority of decapod species are collected, the use of more than one sampling method is recommended.
\end{abstract}

KEYWORDS. Decapoda, Palaemonidae, Atyidae, Grapsidae, ecology.

RESUMO. Durante os anos 2000 e 2001 foram realizadas coletas sazonais de crustáceos decápodes em dois pontos de um rio na Estação Ecológica Juréia-Itatins, utilizando-se duas metodologias distintas (peneira e armadilha). Comparando-se ambos os métodos de coleta, foram constatadas diferenças quanto à composição e riqueza da carcinofauna capturada, grau de dominância e equitatividade, bem como tamanho e número de indivíduos coletados. Além disso, as razões sexuais de Macrobrachium acanthurus (Wiegmann, 1836) podem diferir dependendo do método de coleta utilizado. Devido à grande variedade de hábitats e hábitos dos crustáceos, pode-se inferir que, para um levantamento qualitativo e/ou quantitativo eficaz e apurado da comunidade de crustáceos decápodes dulcícolas de um determinado rio, é recomendado o uso de mais de um método de coleta.

PALAVRAS-CHAVE. Decapoda, Palaemonidae, Atyidae, Grapsidae, ecologia.

Os crustáceos habitam uma grande variedade de hábitats marinhos, dulcícolas e terrestres, nos quais vivem em vários micro-hábitats, desempenhando diversas funções existentes na cadeia trófica. No ambiente de água doce, por exemplo, existe uma zonação da fauna de crustáceos decápodes relacionada a características ambientais (temperatura da água, velocidade da corrente, composição do leito do rio) e aos vários recursos (detritos orgânicos, matéria animal, matéria vegetal, fitoplâncton, zooplâncton) explorados pelas diferentes espécies ao longo das fases de vida de seus indivíduos (CHACE \& Hobis, 1969; Abele \& Blum, 1977; HobBs \& HaRT, 1982; Bond-BuCKuP \& Buckup, 1989; TeixeIRA \& SÁ, 1998; MÜLlER et al., 1999).

Devido a esta heterogeneidade de hábitats e nichos, há uma variedade de métodos de captura para esses animais, entre os quais se destacam armadilhas tipo covo e pitfall, peneiras, puçás, redes, coleta manual e choque elétrico (ABELE \& Blum, 1977; Fievet et al., 1996; CREWE et al., 2001; GARCIA et al., 2003; JohnSON, 2003; Rocha \& Bueno, 2004; Mossolin et al., 2006).

Comparações entre metodologias de coleta são importantes para elucidar questões referentes às metodologias de captura mais eficientes para determinado grupo zoológico, fornecer informações sobre o tipo de fauna amostrada em cada uma das metodologias, à abundância relativa, à proporção e tamanho dos indivíduos, ou ainda detectar vícios de amostragem (Montague, 1980; Fievet et al., 1996; Mayfield et al., 1999; Hyvönen \& Nummi, 2000; Crewe et. al., 2001; GARCiA et al., 2003; JoHNSON, 2003).
Diferenças na proporção de indivíduos e composição faunística relacionadas a diferentes metodologias de coleta já foram enfatizadas por outros autores. HyvöNen \& NumMi (2000) demonstraram que a proporção de invertebrados (principalmente, Chironomidae, Bivalvia, Cladocera e Dytiscidae) de água doce capturada em lagos da Finlândia varia conforme o método de coleta empregado. Garcia et al. (2003) verificaram que existe diferenças significativas no número total de indivíduos, número total de jovens e adultos e número de machos e fêmeas de talassinídeos, dependendo da metodologia de captura utilizada. Alves \& STRIXINo (2003) verificaram que a estrutura e composição da comunidade macrobentônica obtida através de bandejas de espera e pegadores Ekman apresentaram pouca similaridade e diferentes densidades e abundâncias, sugerindo que tais métodos de coleta gerariam informações diferentes sobre a estrutura da biota de um lago em São Paulo. Da mesma forma, Silva et al. (2005) compararam a eficiência de amostradores qualitativos e quantitativos na amostragem da fauna macrobêntonica do rio das Almas, em Goiás, concluindo que as técnicas de coleta qualitativas utilizadas subestimaram a riqueza e a composição faunística da comunidade. Dentre os estudos com crustáceos pode-se destacar Bueno et al. (2003) que concluíram que o amostrador Surber não é adequado para amostrar crustáceos anomuros do gênero Aegla Leach, 1820 presentes em grande quantidade em arroios da região do município de São Francisco de Paula, Rio Grande do Sul. 
O objetivo deste trabalho é demonstrar que duas metodologias largamente utilizadas na captura de crustáceos decápodes de água doce (peneira e armadilhas) amostram indivíduos com hábitos de vida distintos, que vivem em diferentes micro-hábitats, em proporções sexuais e tamanhos diferentes e que podem ou não ser da mesma espécie.

\section{MATERIAL E MÉTODOS}

As espécies utilizadas neste trabalho foram obtidas em dois pontos de um rio de nome desconhecido, na Estação Ecológica Juréia-Itatins (Fig. 1). Ambos os pontos pertencem a um mesmo rio e estão separados por aproximadamente $200 \mathrm{~m}$. Apesar da curta distância que os separa, eles apresentavam micro-hábitats muito distintos e por esta razão foram selecionados. O primeiro ponto (24²3'09' S, 4701'07'”W) possuía substrato rochoso, forte correnteza e profundidade não ultrapassando $50 \mathrm{~cm}$; em alguns locais onde o fluxo de água era menos intenso, formavam-se remansos onde se acumulava grande quantidade de matéria orgânica (principalmente folhas); nas margens havia Mata Atlântica, com grande variabilidade de estratos, mas não havia vegetação marginal parcialmente submersa. No segundo ponto $\left(24^{\circ} 23^{\prime} 05^{\prime}\right.$ 'S, $\left.47^{\circ} 01^{\prime} 08^{\prime \prime} \mathrm{W}\right)$ o substrato era arenoso, a correnteza mais fraca e havia profundidades que chegavam a 1,20 m. Em alguns pontos do leito do rio acumulava-se grande quantidade de matéria vegetal em decomposição, advinda da Mata Atlântica que o margeava; nas margens havia grande acúmulo de vegetação parcialmente submersa.

As coletas foram realizadas sazonalmente, preferencialmente na metade da estação, durante os anos 2000 e 2001. Ao todo foram realizadas oito amostragens em cada um dos pontos de coleta, utilizando-se peneiras e armadilhas para capturar os crustáceos decápodes. No ponto 1 , os crustáceos escondidos debaixo de pedras e seixos no leito do rio eram inicialmente desalojados (remoção do abrigo) e em seguida capturados com a peneira. Já no ponto 2, onde havia vegetação marginal, a peneira era passada vigorosa e rapidamente em

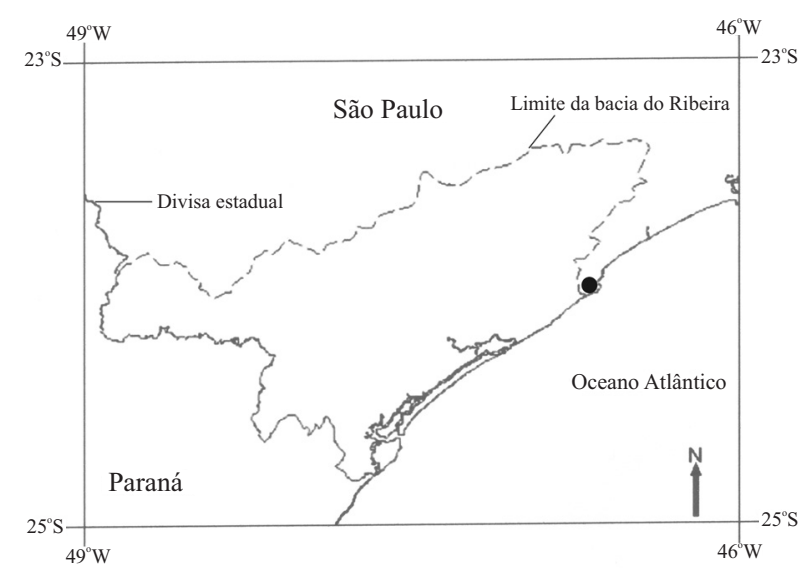

Figura 1. Mapa do sul do estado de São Paulo, com destaque à bacia hidrográfica do Ribeira de Iguape e localização dos pontos de coleta (ponto preto) na Estação Ecológica Juréia-Itatins, SP. movimentos ascendentes por entre a vegetação marginal parcialmente submersa. Além disso, em ambos os pontos, amostras de folhas acumuladas eram rapidamente coletadas para posterior triagem dos crustáceos em bandejas no próprio local. A fim de padronizar o esforço de captura durante as amostragens com peneiras foi estabelecida a utilização de um coletor durante $30 \mathrm{~min}$.

No segundo método, cinco armadilhas do tipo covo (dimensões $25 \mathrm{~cm}$ x $50 \mathrm{~cm}$ x $20 \mathrm{~cm}$ ), iscadas com corações de frango, foram distribuídas aleatoriamente em cada ponto de amostragem no final do período da tarde de um dia e recolhidas nas primeiras horas da manhã do dia seguinte. Ao todo as armadilhas permaneceram submersas por um período de aproximadamente $14 \mathrm{~h}$ em cada uma das oito amostragens realizadas.

Os animais capturados eram imediatamente fixados em álcool $70 \%$ e acondicionados em frascos plásticos. Em laboratório, os animais foram identificados com base nas chaves dicotômicas de VILLALOBOs (1959) e HoBBs \& HART (1982) para camarões carídeos da família Atyidae; Holthuis (1952) para camarões carídeos da família Palaemoidae; e Melo (1996) para caranguejos Grapsidae. Além destes trabalhos, foram consultadas outras publicações (Genofre \& Lobão, 1978; Kensley \& WALKER, 1982; Melo et al., 1988; Ostrovski et al., 1996) contendo descrições de novas espécies não contempladas nas chaves de identificação dos trabalhos mencionados acima. Lotes representativos das espécies coletadas foram depositados no Museu de Zoologia da Universidade de São Paulo e os respectivos números de registro estão disponíveis em RochA \& BuENo (2004).

Um paquímetro digital (precisão $0,01 \mathrm{~mm}$ ) foi utilizado para registrar o tamanho dos animais capturados. Para as espécies de camarões de maior porte, como palemonídeos e atiídeos, considerou-se o comprimento da carapaça (da margem pós-orbital até a margem posterior da carapaça); no caso de espécies de camarões de menor porte, como aqueles do gênero Potimirim Holthuis, 1954, optou-se por medir o comprimento total (da ponta do rostro até a margem posterior do télson); no caso de caranguejos mediu-se a maior largura da carapaça.

Todos os exemplares coletados tiveram o sexo determinado com base em caracteres sexuais específicos. Em camarões carídeos observou-se a presença (machos) ou ausência (fêmeas) do apêndice masculino, localizado no segundo par de pleópodes. A condição ovígera também serviu para o reconhecimento imediato de fêmeas em camarões carídeos. No caso dos caranguejos, os critérios utilizados foram: o formato do abdômen (arredondado nas fêmeas e mais estreito nos machos) e a presença de gonópodes (machos) ou pleópodes (fêmeas) na região abdominal (BuCKuP \& BOND-BuCKUP, 1999).

Os dois métodos de coleta descritos foram comparados, quanto a aspectos ecológicos (riqueza, dominância, equitatividade), proporções entre machos e fêmeas e tamanho dos indivíduos coletados. Para verificar diferenças significativas entre as proporções de machos e fêmeas em relação ao método de coleta utilizou-se o teste $\chi^{2}$ (qui-quadrado). Para a comparação estatística do 
tamanho dos indivíduos, foi aplicado o teste de MannWhitney (uma vez que o teste de Shapiro-Wilk não detectou distribuição normal nas amostras comparadas) calculado através do programa PAST (HAMMER et al., 2001), o qual também foi utilizado para o cálculo dos índices de riqueza, dominância e equitatividade. Espécies de decápodes capturadas pelos dois métodos de coleta e em número minimamente suficiente para a realização de análises estatísticas foram selecionadas para a comparação de tamanho e proporção sexual por método de coleta. Todas as análises estatísticas foram baseadas em ZAR (1996) e realizadas com nível de significância de $95 \%$.

\section{RESULTADOS}

No ponto 1 , a coleta feita com peneira amostrou quatro espécies de camarões, duas delas sendo da família Atyidae, Atya scabra Leach, 1815 e Potimirim glabra (Kingsley, 1878), e outras duas da família Palaemonidae, M. acanthurus (Wiegmann, 1836) e M. olfersi (Wiegmann, 1836). Por outro lado, nas coletas utilizando-se armadilhas houve captura de três espécies de palemonídeos, $M$. acanthurus, M. olfersi e Macrobrachium carcinus (Linnaeus, 1758) (Tab. I).

Já no ponto 2 foram capturados exemplares dos camarões $M$. acanthurus, M. olfersi, Palaemon pandaliformis (Stimpson, 1871), Potimirim glabra e do caranguejo Pachygrapsus transversus (Gibbes, 1850) em coletas realizadas com peneira, enquanto que, com o uso de armadilhas, foram amostrados somente exemplares de M. acanthurus, $M$. olfersi e Palaemon pandaliformis (Tab. II).
No ponto 1, as espécies dominantes nas amostragens com peneiras e armadilhas foram $P$. glabra e M. olfersi, respectivamente (Fig. 2). Para o ponto 2, a espécie dominante nas amostras envolvendo ambas as metodologias foi M. acanthurus (Fig. 3)

A riqueza de espécies capturadas foi maior nas amostras realizadas com peneiras, tanto para o ponto 1 como para o ponto 2 (Tab. III). Os graus de dominância calculado para as coletas realizadas no ponto $1 \mathrm{com}$ peneiras e armadilhas foram, respectivamente, 0,855 e 0,634 . Para o ponto 2 , os graus de dominância calculados foram 0,425 em coletas com peneira e 0,647 em coletas com armadilhas. Por outro lado, os graus de equitatividade das amostras com peneira e armadilha no ponto 1 foram, respectivamente, 0,3551 e 0,5918; para o ponto 2 os valores foram 0,5922 e 0,6294 em coletas feitas em peneira e armadilha, respectivamente.

O teste de Mann-Whitney detectou diferenças significativas entre o tamanho dos indivíduos de $M$. acanthurus e de $M$. olfersi coletados com peneira e armadilha, tanto no ponto 1 como no ponto 2 (Tab. IV). Portanto, os exemplares capturados com peneira exibiam tamanhos inferiores em relação àqueles capturados com armadilhas.

Apenas as amostras de $M$. acanthurus do ponto 2 apresentaram número suficiente de dados para a verificação de diferenças significativas na proporção de machos e fêmeas capturados com peneira e armadilhas. Dessa forma, o teste de qui-quadrado detectou diferença significativa na razão sexual dessa espécie tanto em amostras coletadas com peneira (razão sexual: $0,6: 1 ; \chi^{2}=$ $7,04 ; 0,01<\mathrm{p}<0,005)$ como naquelas utilizando-se armadilhas (razão sexual: $1,8: 1 ; \chi^{2}=10,96 ; \mathrm{p}<0,001$ ).

Tabela I. Espécies coletadas com peneira e armadilha em rio sem nome (ponto 1) (24²3'09'”S, 4701'07'W) da Estação Ecológica Juréia-Itatins, SP (M, machos; F, fêmeas; N total, número total de indivíduos; T.M. (M), tamanho médio dos machos em milímetros ( \pm desvio padrão); T.M. (F), tamanho médio das fêmeas em milímetros ( \pm desvio padrão)).

\begin{tabular}{|c|c|c|c|c|c|c|}
\hline Método de coleta & Espécie & M & $\mathrm{F}$ & $\mathrm{N}$ total & T.M. (M) & T.M. (F) \\
\hline \multirow[t]{4}{*}{ Peneira } & Atya scabra & 8 & 11 & 19 & $13,6( \pm 4,2)$ & $11,5( \pm 3,4)$ \\
\hline & Potimirim glabra & 563 & 258 & 821 & $14,0( \pm 1,4)$ & $17,2( \pm 2,2)$ \\
\hline & Macrobrachium olfersi & 11 & 21 & 32 & $12,4( \pm 5,0)$ & $10,7( \pm 2,4)$ \\
\hline & Macrobrachium acanthurus & 10 & 7 & 17 & $11,0( \pm 5,3)$ & $9,6( \pm 1,4)$ \\
\hline \multirow[t]{3}{*}{ Armadilha } & Macrobrachium acanthurus & 34 & 12 & 46 & $18,6( \pm 8,7)$ & $10,0( \pm 2,7)$ \\
\hline & Macrobrachium olfersi & 65 & 85 & 150 & $14,7( \pm 4,0)$ & $11,9( \pm 2,3)$ \\
\hline & Macrobrachium carcinus & 0 & 1 & 1 & & 31,2 \\
\hline
\end{tabular}

Tabela II. Espécies coletadas com peneira e armadilha em rio sem nome (ponto 2) (24²3’05' S, 4701'08'’W) da Estação Ecológica Juréia-Itatins, SP (M, machos; F, fêmeas; N total, número total de indivíduos; T.M. (M), tamanho médio dos machos em milímetros ( \pm desvio padrão); T.M. (F), tamanho médio das fêmeas em milímetros ( \pm desvio padrão)).

\begin{tabular}{|c|c|c|c|c|c|c|}
\hline Método de coleta & Espécie & $\mathrm{M}$ & $\mathrm{F}$ & $\mathrm{N}$ total & T.M. (M) & T.M. (F) \\
\hline \multirow[t]{5}{*}{ Peneira } & Macrobrachium acanthurus & 49 & 79 & 128 & $8,3( \pm 3,8)$ & $8,7( \pm 2,7)$ \\
\hline & Macrobrachium olfersi & 4 & 15 & 19 & $10,45( \pm 2,11)$ & $11,12( \pm 2,12)$ \\
\hline & Palaemon pandaliformis & 3 & 5 & 8 & $4,81( \pm 1,06)$ & $6,36( \pm 1,38)$ \\
\hline & Pachygrapsus tranversus & 3 & 2 & 5 & $17,93( \pm 4,88)$ & $10,25( \pm 1,20)$ \\
\hline & Potimirim glabra & 23 & 35 & 58 & $14,67( \pm 2,76)$ & $17,27( \pm 3,82)$ \\
\hline \multirow[t]{3}{*}{ Armadilha } & Macrobrachium acanthurus & 81 & 44 & 125 & $16,9( \pm 7,2)$ & $13,0( \pm 4,3)$ \\
\hline & Macrobrachium olfersi & 5 & 3 & 8 & $16,7( \pm 4,85)$ & $12,17( \pm 0,23)$ \\
\hline & Palaemon pandaliformis & 11 & 15 & 26 & $4,90( \pm 0,56)$ & $5,44( \pm 0,48)$ \\
\hline
\end{tabular}




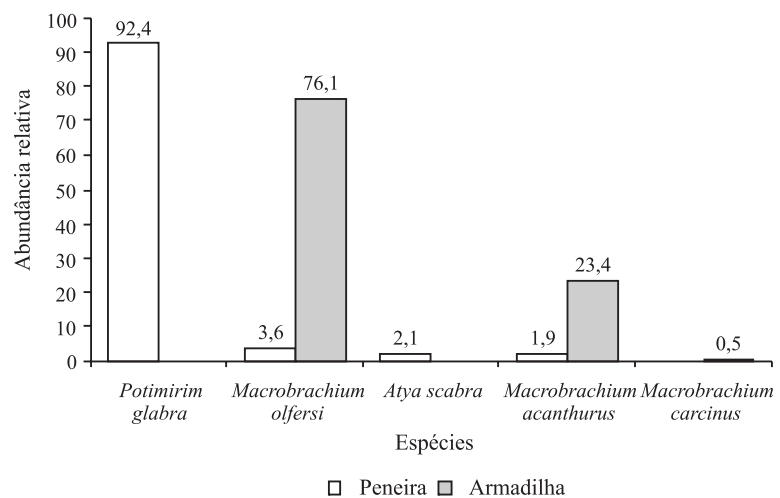

Figura 2. Abundância relativa das espécies de crustáceos capturados com peneira e armadilha no ponto 1 (24⒉ $3^{\prime} 09^{\prime}$ 'S, $47^{\circ} 01^{\prime} 07^{\prime}$ 'W) de um rio sem nome da Estação Ecológica JuréiaItatins, SP.

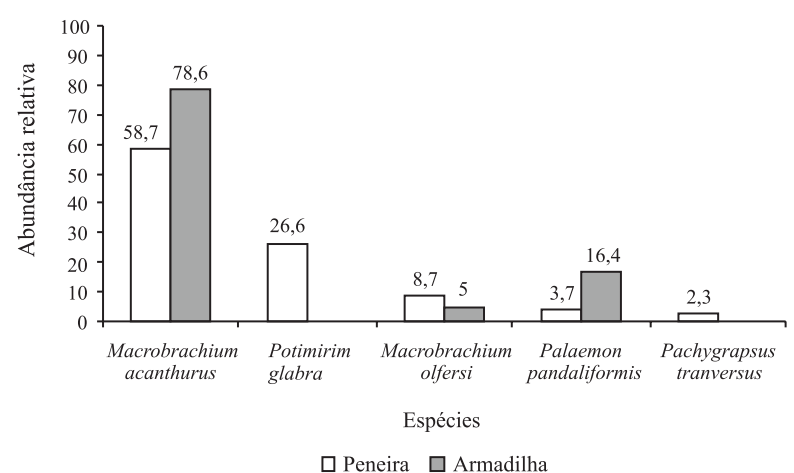

Figura 3. Abundância relativa das espécies de crustáceos capturados com peneira e armadilha no ponto $2\left(24^{\circ} 23^{\prime} 05^{\prime}\right.$ ' $\mathrm{S}$, $\left.47^{\circ} 01^{\prime} 08^{\prime \prime} \mathrm{W}\right)$ de um rio sem nome da Estação Ecológica JuréiaItatins, SP.

Tabela III. Índices de riqueza, dominância e equitatividade calculados

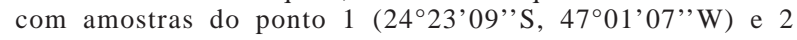
(242' $05^{\prime}$ 'S $\left.44^{\circ} 01^{\prime} 08^{\prime \prime} \mathrm{W}\right)$ da Estação Ecológica Juréia-Itatins, SP.

\begin{tabular}{lcccc}
\hline & \multicolumn{2}{c}{ Ponto 1 } & \multicolumn{2}{c}{ Ponto 2 } \\
\hline Índices & Peneira & Armadilha & Peneira & Armadilha \\
\hline Riqueza & 4 & 3 & 5 & 3 \\
Dominância & 0,855 & 0,6343 & 0,425 & 0,6473 \\
Equitatividade & 0,3551 & 0,5918 & 0,5922 & 0,6294
\end{tabular}

\section{DISCUSSÃO}

Os atíídeos são camarões que se alimentam por filtração ou remoção de material orgânico do substrato mediante o uso dos tufos de cerdas localizados no primeiro e segundo pares de pereópodes (HOBBS \& HART, 1982). Especificamente no caso das espécies do gênero Atya Leach, 1816, os animais filtram passivamente a água corrente e retiram detritos orgânicos que se encontram no leito do rio (Felgenhauer \& Abele, 1985). O gênero Potimirim, ao contrário, tem seus quelípodes com cerdas especializadas para uma alimentação mais ativa, que consiste do perifiton, detritos, algas e até mesmo exúvias de insetos (Fryer, 1977; НоbBs \& Hart, 1982; Felgenhauer \& Abele, 1985; Souza \& Moulton, 2005; SouZA et al., 2007).

$\mathrm{O}$ fato dos atiídeos terem sua dieta baseada em detritos ou pequenas partículas em suspensão na água torna as armadilhas ineficazes para sua captura, uma vez que estas são um método passivo de coleta, necessitando de uma isca para atrair os animais. Por outro lado, essa metodologia de coleta é eficaz para crustáceos carnívoros ou onívoros, como é o caso dos camarões palemonídeos (ABele \& Blum, 1977), que podem ser atraídos pela isca utilizada (neste caso, corações de frango).

Além disso, o gênero Potimirim é conhecido por habitar a vegetação marginal submersa e o folhiço acumulado no fundo de remansos dos rios, enquanto Atya esconde-se sob as pedras dos leitos de rios com forte correnteza e águas limpas (Muller, 1892; SMALleY, 1963; Abele \& Blum, 1977; Нobbs \& Hart, 1982; Felgenhauer \& Abele, 1985; Barros \& Fontoura, 1996a, b; Lima \& Oshiro, 1999; Pyron et al., 1999; Galvão \& Bueno, 2000; Lima et al., 2006); estes são hábitats-alvo da peneira, que por se tratar de uma metodologia ativa de coleta, desaloja e captura esses animais. Ainda com base no tipo de hábitat em que vivem os camarões do gênero Atya, pode-se inferir que as características físicas do ponto 2, cujo leito era arenoso e a correnteza fraca, seriam fatores que justificariam a ausência de camarões A. scabra nas amostras dessa localidade.

A não captura de $M$. carcinus em coletas com peneira no ponto 1 pode estar relacionada com o próprio comportamento desta espécie cujos adultos se escondem em fendas e buracos entre grandes pedras ou sob troncos

Tabela IV. Resultados dos testes de comparação (Mann-Whitney) entre o tamanho dos indivíduos capturados em peneira e armadilha, nos pontos 1 e 2 de um rio de nome desconhecido da Estação Ecológica Juréia-Itatins, SP.

\begin{tabular}{lcccc}
\hline Local & Espécie & Método de Coleta & Tamanho dos indivíduos $(\mathrm{mm})$ & Teste de Mann-Whitney \\
\hline Ponto 1 & Macrobrachium acanthurus & Peneira & $6,6-23,1 /$ média $=10,40( \pm 4,11)$ & $\mathrm{U}=220 ; \mathrm{p}=0,008288$ \\
& \multirow{2}{*}{ Macrobrachium olfersi } & Armadilha & $4,8-33,4 /$ média $=16,36( \pm 8,52)$ & \\
& & Peneira & $6,0-23,7 /$ média $=11,30( \pm 3,53)$ & $\mathrm{U}=1645 ; \mathrm{p}=0,005994$ \\
& Armadilha & $5,3-21,9 /$ média $=13,10( \pm 3,37)$ & \\
Ponto 2 & Macrobrachium acanthurus & Peneira & $3,5-28,8 /$ média $=8,74( \pm 3,61)$ & $\mathrm{U}=3012 ; \mathrm{p}<<0,001$ \\
& \multirow{2}{*}{ Macrobrachium olfersi } & Armadilha & $4,4-31,4 /$ média $=15,49( \pm 6,69)$ & $\mathrm{U}=26,5 ; \mathrm{p}=0,009272$ \\
& & Peneira & $5,6-14,7 /$ média $=10,98( \pm 2,07)$ & $\mathrm{C}$
\end{tabular}


caídos, geralmente em partes mais profundas de riachos de água doce ou salobra (CHACE \& HoвBs, 1969; GAмBA, 1982; LoBÃo et al., 1986; BOND-BUCKUP \& BUCKUP, 1989). Por outro lado, o hábito noturno e onívoro de $M$. carcinus (LEwIs et al., 1966) foram fatores preponderantes para que esta espécie fosse capturada pelas armadilhas que operam durante a noite, justamente quando os indivíduos deixam seus abrigos para se alimentar, podendo ser atraídos pela isca.

É provável que os adultos de $M$. carcinus não tenham sido capturados no ponto 2 , tanto em coletas com peneiras como em armadilhas, pois nesse ponto o fundo do rio era de areia, sem pedras ou troncos que serviriam de abrigo para os indivíduos. Pyron et al. (1999) estudaram a influência de restos orgânicos (madeira, folhas mortas, etc.) na distribuição de camarões em remansos de um riacho em Porto Rico e concluíram que o aumento na quantidade de troncos de árvore não influenciou significativamente a abundância de camarões predadores, como $M$. carcinus. Entretanto, os autores enfatizaram que os remansos estudados já apresentavam abrigos em abundância, na forma, principalmente, de fendas em rochas e que a adição de galhos e outros restos de madeira não alteraram de maneira significativa a estrutura pré-existente dos locais de estudo. Além disso, os resultados obtidos para as regiões de remansos não se aplicam ao canal central do rio, onde galhadas e outros restos vegetais desempenham um papel fundamental como estruturas de refúgio para a carcinofauna (PYRON et al., 1999).

Segundo Lewis et al. (1966) os jovens de $M$. carcinus preferem habitar riachos com fundo de lama ou areia; este tipo de fundo não é característico do ponto 1 , onde o leito era formado essencialmente por seixos de rocha, o que justificaria a ausência de jovens nas amostras desta localidade. No caso do ponto 2, cujo fundo era de areia, é possível que a realização de coletas sazonais tenha impossibilitado a amostragem dos jovens de $M$. carcinus, ou ainda que a metodologia de coleta não tenha sido eficiente para capturar esses indivíduos. LEWIS et al. (1966) amostraram formas jovens dessa espécie em um rio na ilha de St. James, Barbados, utilizando puçás.

Pachygrapsus transversus foi coletado com peneira no ponto 2 devido à proximidade deste local com a foz do rio e os costões rochosos litorâneos. FuRTADo-OGAwA (1977) relata que essa espécie é eurihalina, podendo ser encontrada desde o infra até o supra-litoral. Portanto, é possível que os exemplares capturados fossem habitantes do supra-litoral e estivessem realizando incursões na região de coleta e foram capturados acidentalmente entre a vegetação marginal.

Palaemon pandaliformis é encontrado em ambiente de água doce e estuarino. Costa et al. (2000) relataram a captura desta espécie também em ambiente marinho (Baía de Ubatuba, SP). De acordo com MülLER et al. (1996) e Lima \& OshiRo (2002a), exemplares de $P$. pandaliformis podem ser capturados com o auxílio de peneira ou puçá, passando-os por entre a vegetação marginal parcialmente submersa ou utilizando-os para recolher amostras de folhiço acumulado em áreas mais calmas no fundo do leito do rio. No presente estudo, $P$. pandaliformis foi mais abundante em coletas que utilizaram armadilhas, demonstrando que essa metodologia de coleta também é eficaz para amostrar essa espécie.

As espécies do gênero Potimirim são muito abundantes nos rios em que ocorrem, constituindo populações extremamente numerosas (BARROS \& FonTOURA, 1996a; LIMA \& OshIRO, 2002b; LimA et al., 2006). No presente estudo, $P$. glabra foi capturada em número muito superior ( $\mathrm{n}=821$ ou $92,4 \%$ da amostra) se comparada às demais espécies encontradas no ponto 1 . Tal fato exerceu influência direta no valor do grau de dominância nas coletas com peneira que foi maior que aquele calculado para as amostras realizadas com armadilhas, nas quais a referida espécie não foi coletada. Por outro lado, a presença de duas espécies predominantes ( $M$. acanthurus $58,7 \%$ e $P$. glabra $26,6 \%$ ) nas coletas com peneira do ponto 2 acabou por influenciar o valor do grau de dominância nessa amostra, fazendo-o ficar abaixo daquele encontrado para as coletas com armadilhas, nas quais $M$. acanthurus predominou $(78,6 \%)$ fortemente em relação às demais espécies, sem a presença de P. glabra.

Segundo Smalley (1963), P. glabra prefere habitar riachos rasos, com fundo de pedras e folhiço acumulado. Dessa forma, é provável que essa espécie tenha ocorrido de maneira menos abundante no ponto 2 , em relação ao ponto 1 , devido à ausência desse tipo de ambiente.

As diferenças significativas encontradas na comparação entre os tamanhos de $M$. acanthurus e $M$. olfersi, capturados com peneira e armadilha, podem ser explicadas mediante análise do comportamento dos animais em suas diferentes fases de vida. Jovens de palemonídeos habitam, principalmente, pedras de pequeno e médio porte, o folhiço acumulado junto ao fundo do rio e a vegetação marginal; locais amostrados pela peneira. Os indivíduos maiores destes camarões são capturados pelas armadilhas, pois tendem a se entocar em locais mais profundos, sob troncos ou pedras grandes, que não podem ser virados manualmente, saindo à noite para se alimentar; justamente o período em que as armadilhas operam. Da mesma forma, PYRON et al. (1999) concluíram que camarões jovens do gênero Macrobrachium Bate, 1868 estavam presentes em locais com folhiço e fundo de areia, enquanto os adultos eram mais abundantes em remansos mais profundos, com leito pedregoso e fendas entre os seixos.

Os resultados obtidos no presente trabalho mostram que a razão sexual de $M$. acanthurus coletada no ponto 2 difere dependendo do método de coleta utilizado. Nota-se que a razão sexual das coletas com peneiras foi deslocada em favor das fêmeas, enquanto a razão sexual das coletas com armadilhas foi deslocada em favor dos machos. Dessa forma, a metodologia de coleta poderia estar influenciando a amostragem neste caso, uma vez que se houvesse nessa referida população um deslocamento da razão sexual em favor de qualquer um dos sexos, seria esperado que ambas as metodologias de coleta demonstrassem o mesmo padrão. MonTOYA (2003) encontrou maior proporção de fêmeas e grande número de jovens de três espécies de Macrobrachium associadas a raízes de Eichhornia crassipes (Pontederiaceae), sugerindo que essas espécies utilizam 
essas macrófitas como locais de abrigo e reprodução. Neste sentido, é possível que as fêmeas de $M$. acanthurus coletadas no ponto 2 apresentem essa mesma estratégia, concentrando-se na vegetação marginal, ficando mais acessíveis às coletas com peneiras. No entanto, mais estudos fazem-se necessários no sentido de esclarecer essa possível influência da metodologia sobre a razão sexual em camarões palemonídeos, uma vez que vários fatores tais como migrações sazonais, mortalidade, longevidade, predação, podem influenciar a proporção sexual de crustáceos (WENNER, 1972).

Devido à grande variedade de hábitats e hábitos dos crustáceos decápodes dulcícolas, conclui-se que para um levantamento mais eficaz e apurado destes animais, em um determinado rio, é necessário o uso de mais de um método de coleta, a fim de amostrar todas as espécies que possam estar presentes. Da mesma forma, num estudo quantitativo, o uso dos dois métodos de coleta torna-se interessante, pois permite amostrar um universo maior de classes de tamanho e, em alguns casos, razões sexuais diferenciadas, relacionados diretamente aos micro-hábitats focados por cada um dos métodos. Contudo, é necessário cautela durante a análise dos dados, uma vez que o esforço de coleta pode diferir em relação a cada metodologia empregada. Portanto não é recomendada a união dos dados para uma só análise quando relacionada à estrutura populacional ou estabelecimento de coortes. Análises distintas, método a método, permitem resultados mais minuciosos, possibilitando discussões mais aprofundadas e detalhadas sobre uma determinada população.

Agradecimentos. O autor agradece ao Prof. Dr. Sergio Luiz de Siqueira Bueno (USP) pela orientação durante a execução e elaboração da dissertação de Mestrado que resultou neste trabalho. Aos Drs. Fábio Kyiohara e Emerson C. Mossolin pelo auxílio durante as atividades de campo. Ao Prof. Dr. Roberto Munehisa Shimizu (USP) pelo auxílio nas análises estatísticas. À Profa. Dra. Georgina Bond-Buckup (UFRGS) e ao Prof. Dr. Célio U. Magalhães (INPA), que confirmaram a identificação dos exemplares. À FAPESP (Fundação de Amparo à Pesquisa do Estado de São Paulo) pela concessão de auxílio financeiro (Programa BIOTA-FAPESP, processo ${ }^{\circ}$ 98/05073-4) e bolsa de Mestrado (processo $n^{\circ}$ 00/ 02119-5). À Coordenação do Programa de Pós-graduação em Zoologia e Departamento de Zoologia do IB/USP, pelo apoio logístico e finalmente aos funcionários da Estação Ecológica JuréiaItatins, bem como do Instituto Florestal do Estado de São Paulo.

\section{REFERÊNCIAS}

Abele, L. G. \& Blum, N. 1977. Ecological aspects of the freshwater decapod crustaceans of the Pelas archipelago, Panamá. Biotropica 9(4):239-252.

Alves, R. G. \& Strixino, G. 2003. The sampling of benthic macroinvertebrates using two different methods: waiting trays and Ekman collector. Acta Limnologica Brasiliense 15(3): $1-6$.

Barros, M. P. \& Fontoura, N. F. 1996a. Biologia reprodutiva de Potimirim glabra (Kingsley, 1878) (Crustacea, Decapoda, Atyidae), na praia da Vigia, Garopaba, Santa Catarina, Brasil. Naupilus 4:1-10.

1996b. Crescimento de Potimirim glabra (Kingsley, 1878) (Crustacea, Decapoda, Atyidae), na praia da Vigia, Garopaba, Santa Catarina, Brasil. Naupilus 4:11-28.

Bond-Buckup, G. \& Buckup, L. 1989. Os Palaemonidae de águas continentais do Brasil meridional (Crustacea, Decapoda). Revista Brasileira de Biologia 49(4):883-896.

Buckup, L. \& Bond-Buckup, G. 1999. Os crustáceos do Rio Grande do Sul. Porto Alegre, Ed. Universidade / UFRGS. 503 p.
Bueno, A. A. P.; Bond-Buckup, G. \& Ferreira, B. D. P. 2003. Estrutura da comunidade de invertebrados bentônicos em dois cursos d'água do Rio Grande do Sul, Brasil. Revista Brasileira de Zoologia 20(1):115-125.

Chace JR., F. A. \& HobBs, H. H. 1969. The freshwater and terrestrial decapod crustaceans of the West Indies with special reference to Dominica. United States National Museum Bulletin 292:1-256.

Costa, R. C.; Fransozo, A.; Mantelatto, F. L. M. \& Castro, R. H 2000. Occurrence of shrimp species (Crustacea: Decapoda: Natantia: Penaeidea and Caridea) in Ubatuba, SP, Brazil. Proceedings of the Biological Society of Washington, 113(3):776-781

Crewe, T. L.; Hamilton, D. J. \& Diamond, A. W. 2001. Effects of mesh size on sieved samples of Corophium volutator. Estuarine, Coastal and Shelf Science 53(2):151-154.

Felgenhauer, B. E. \& Abele, L. G. 1985. Feeding structures of two Atyid shrimps with comments on caridean phylogeny. Journal of Crustacean Biology 5(3):397-419.

Fievet, E.; Demorais, L. T. \& Demorais, A. T. 1996. Quantitative sampling of freshwater shrimps - comparison of two electrofishing procedures in a Caribean stream. Hydrobiologia 138:273-287.

FrYER, G. 1977. Studies on the funcional morphology and ecology of the Atyid prawns of Dominica. Philosophical Transactions of the Royal Society of London 227(952):57-128.

Furtado-Ogawa, E. 1977. Notas biológicas sobre Pachygrapsus transversus (Gibbes, 1850) no Estado do Ceará (Crustacea: Brachyura). Arquivos de Ciências do Mar 17(2):107-113.

Galvão, R. \& Bueno, S. L. S. 2000. Population structure and reproductive biology of the Camacuto shrimp, Atya scabra (Leach, 1815) (Decapoda, Caridea, Atyidae), from São Sebastião, Brazil. Crustacean Issues 12:291-299.

GambA, A. L. 1982. Macrobrachium: its presence in estuaries of the Northern Venezuelan coast (Decapoda, Palaemonidae). Caribean Journal of Science 18(1-4):23-26.

Garcia, K. E.; Embry, S. J.; Grossblat, D.; Holbrook, A. M.; Mclaren, W. M.; Reed, S. K.; Wildey, H. C. \& Shuster, S. M. 2003. A comparison of two methods for sampling the Gulf of California mud shrimp, Neotrypacea uncinata (Crustacea: Thalassinidea). Journal of Natural History 37:1847-1854.

Genofre, G. S. \& Lobão, V. L. 1978. Macrobrachium holthuisi, sp. n., a new species of shrimp (Decapoda, Macrura). Crustaceana 35(3):273-276.

Hammer, $\varnothing$.; Harper, D. A. T. \& Ryan, P. D. 2001. PAST: Paleontological statistics software package for education and data analisys. Paleontologia Eletronica 4(1):1-9. <http:// palaeo-eletronica.org/2001_1/past/issue1_01.htm>.

Hobbs, H. H. \& Hart, C. W. 1982. The Shrimp genus Atya (Decapoda, Atyidae). Smithsonian Contribution to Zoology 364:1-143.

Holthuis, L. B. 1952. A general revision of the Palaemonidae (Crustacea, Decapoda, Natantia) of the Americas. II. The subfamily Palaemoninae. Occasional Paper of the Allan Hancock Foundation 12:1-396.

Hyvönen, T. \& Nummi, P. 2000. Activity traps and the corer: complementary methods for sampling aquatic invertebrates. Hydrobiologia 432:121-125.

Johnson, P. T. 2003. Biased sex ratios in fiddler crabs (Brachyura, Ocypodidae): a review and evaluation of the influence of sampling method, size class, and sex-specific mortality. Crustaceana 76(5):559-580.

Kensley, B. \& Walker, I. 1982. Palaemonid shrimps from the Amazon Basin, Brazil (Crustacea: Decapoda: Natantia). Smithsonian Contributions to Zoology 362:1-28.

Lewis, J. B.; Ward, J. \& McIver, A. 1966. The breeding cycle, growth and food of the fresh water shrimp Macrobrachium carcinus (Linnaeus). Crustaceana 10(1):48-52.

Lima, G. V. \& Oshiro, L. M. Y. 1999. Aspectos reprodutivos do camarão de água doce Potimirim potimirim (Müller, 1881) (Crustacea, Decapoda, Atyidae) do rio Sahy, Mangaratiba/RJ. Naupilus 7:65-71.

2002a. Aspectos reprodutivos de Palaemon pandaliformis (Stimpson) (Crustacea, Decapoda, Palaemonidae) no rio Sahy, Mangaratiba, Rio de Janeiro, Brasil. Revista Brasileira de Zoologia 19(3):855-860. 
2002b. Aspectos reprodutivos de Potimirim glabra (Kingsley) (Crustacea, Decapoda, Atyidae) no rio Sahy, Mangaratiba, Rio de Janeiro, Brasil. Revista Brasileira de Zoologia 19(3):861-866

Lima, G.; Silveira, C. M. \& Oshiro, L. M. 2006. Estrutura populacional dos camarões simpátricos Potimirim glabra e Potimirim potimirim (Crustacea, Decapoda, Atyidae) no rio Sahy, Rio de Janeiro, Brasil. Iheringia, Série Zoologia 96(1):81-87.

Lobão, V. L.; Valenti, W. C. \& Mello, J. T. C. 1986. Crescimento relativo de Macrobrachium carcinus (L.) (Decapoda, Palaemonidae). Boletim do Instituto de Pesca 13(1):105109.

Maypield, G. M.; Branch, A. C. \& Cockcroft, A. C. 1999. Determining prey availability for rock lobsters, Jasus lalandii: diver-sampling versus monochrome video photography. Marine Biology 135(2):347-352.

Melo, G. A. S. 1996. Manual de identificação dos Brachyura (caranguejos e siris) do litoral brasileiro. São Paulo, Plêiade/FAPESP. 603p.

Melo, G. A. S.; Lobão, V. L. \& Fernandes, W. M. 1988. Redescrição de Macrobrachium birai, Lobão, Melo \& Fernandes e de Macrobrachium petronioi, Melo, Lobão \& Fernandes (Crustacea, Decapoda) palemonídeos da região sul do estado de São Paulo; Brasil. Boletim do Instituto de Pesca 15(1):89-97.

Montague, C. L. 1980. A natural history of temperate western Atlantic fiddler crabs (genus $U c a$ ) with reference to their impact on the salt marsh. Contribution in Marine Science 23:25-54

Montoya, J. V. 2003. Freshwater shrimps of the genus Macrobrachium associated with roots of Eichhornia crassipes (Water Hyacinth) in the Orinoco Delta (Venezuela). Caribean Journal of Science 39(1):155-159.

Mossolin, E. C.; Shimizu, R. M. \& Bueno, S. L. S. 2006. Population structure of Alpheus armillatus (Decapoda, Alpheidae) in São Sebastião and Ilhabela, Southeastern Brazil. Journal of Crustacean Biology 26(1):48-54.

MüLleR, F. 1892. O camarão miudo do Itajahy, Atyoida potimirim. Archivos do Museu Nacional 8:155-178,

Müller, Y. M. R.; Nazari, E. M.; Ammar, D.; Ferreira, E. C.; Beltrame, I. T.; \& Pacheco, C. 1999. Biologia dos Palaemonidae da bacia hidrográfica de Ratones, Florianópolis, SC, Brasil. Revista Brasileira de Zoologia 16(3):629-636.

Müller, Y. M. R.; Nazari, E. M.; Bressan, C. M. \& Ammar, D.
1996. Aspectos da reprodução de Palaemon pandaliformis (Stimpson) (Decapoda, Palaemonidae) no manguezal de Ratones, Florianópolis, Santa Catarina. Revista Brasileira de Zoologia 13(3):633-642.

Ostrovski, M. C.; Fonseca, K. M. L.; Silva-Ferreira, T. C. G. \& Matos, F. J. P. 1996. Macrobrachium denticulatum n. sp. a new species of shrimp from the São Francisco Basin, Northeastern Brazil. Crustaceana 69(3):359-367.

Pyron, M.; Covich, A. P. \& Black, R. W. 1999. On the importance of pool morphology and woody debris to distributions of shrimp in a Puerto Rican headwater stream. Hydrobiologia 405:207-215.

Rocha, S. S. \& Bueno, S. L. S. 2004. Crustáceos decápodes de água doce com ocorrência no Vale do Ribeira de Iguape e rios costeiros adjacentes, São Paulo, Brasil. Revista Brasileira de Zoologia 21(4):1001-1010.

Silva, L. C. F.; Vieira, L. C. G.; Costa, D. A.; Lima Filho, G. F.; Vital, M. V. C.; Carvalho, R. A.; Silveira, A. V. T. \& Oliveira, L. C. 2005. Qualitative and quantitative benthic macroinvertebrate samplers in Cerrado streams: a comparative approach. Acta Limnologica Brasiliense 17(2):123-128.

SouzA, M. L. \& Moulton, T. P. 2005. The effects of shrimps on the benthic material in a Brazilian island stream. Freshwater Biology 50(4):592-602.

Souza, M. L.; Moulton, T. P.; Silveira, R. M. L.; Krsulovié, F. A. M. \& BRito, E. F. 2007. Responses of Chironomidae (Diptera Insecta) to the exclusion of shrimps and Ephemeroptera in a coastal forest stream, Rio de Janeiro, Brazil. Brazilian Journal of Biology 67(1):47-51.

Smalley, A. E. 1963. The genus Potimirim in Central America (Crustacea, Atyidae). Revista de Biologia Tropical 11(2): 177-183.

TeixeIRA, R. \& SÁ, H. S. 1998. Abundância de macrocrustáceos decápodes nas áreas rasas do complexo lagunar Mundaú/ Mangaba, AL. Revista Brasileira de Biologia 58(3):393404

Villalobos, A. 1959. Contribuicion al conocimiento de los Atyidae de Mexico II (Crustacea, Decapoda). Estudio de algunas especies del genero Potimirim (=Ortmania), con descripción de una especie nueva en Brasil. Annales del Instituto de Biologia de la Universidad de México 30:269-330.

WenNer, A. M. 1972. Sex ratio as a function of size in marine Crustacea. The American Naturalist 106(949):321-350.

ZAR, J. H. 1996. Biostatistical analysis. 3. ed. New Jersey, Prentice Hall. 662p.

Recebido em julho de 2009. Aceito em fevereiro de 2010. ISSN 0073-4721

Artigo disponível em: www.scielo.br/isz 